
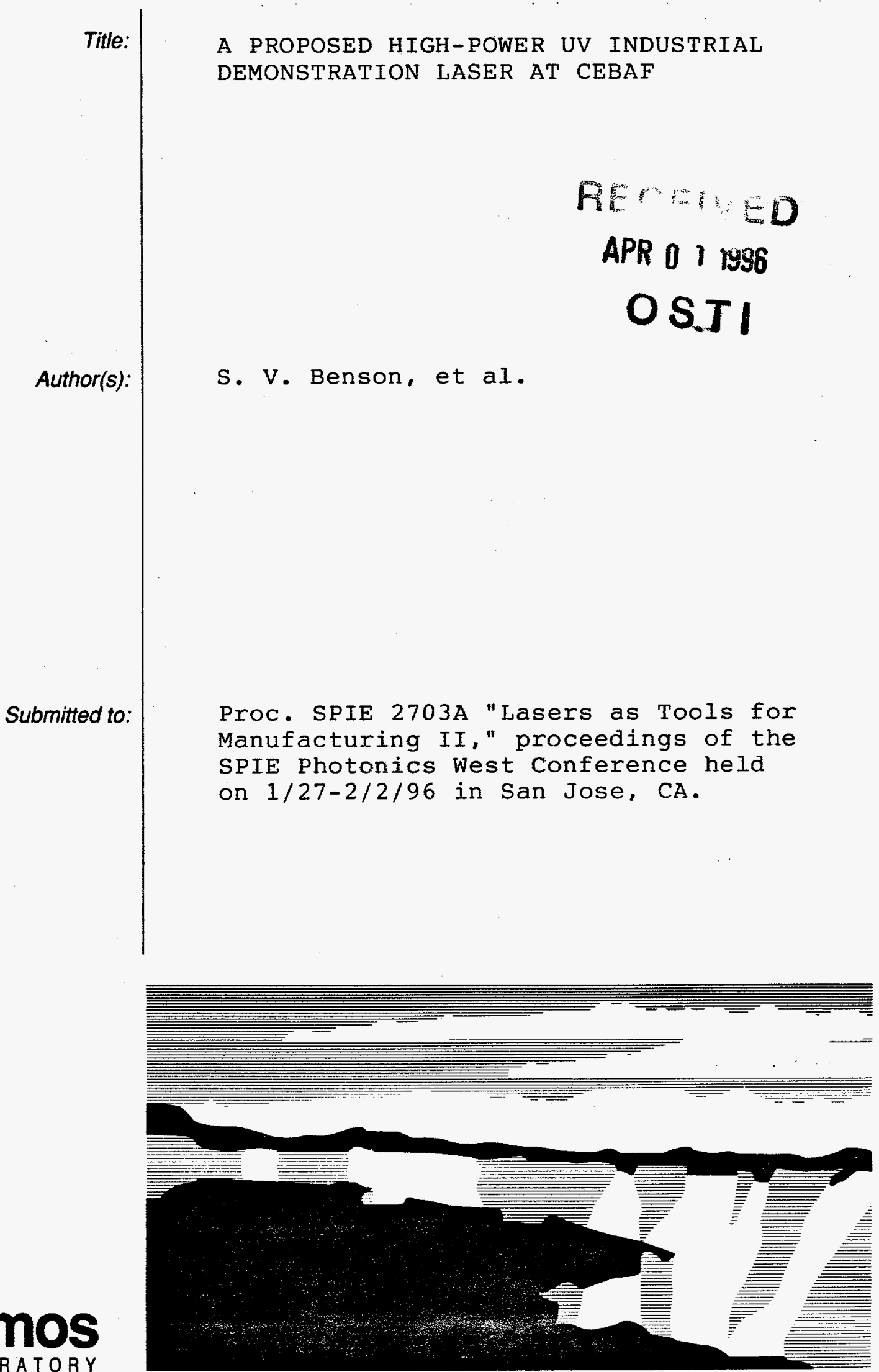

Los Alamos National Laboratory, an affirmative action/equal opportunity emplóyer, is operated by the University of California for the U.S. Department of Energy under contract W-7405-ENG-36. By acceptance of this article, the publisher recognizes that the U.S. Government retains a nonexclusive, royalty-free license to publish or reproduce the published form of this contribution, or to allow others to do so, for U.S. Government purposes. The Los Alamos National Laboratory requests that the publisher identity this article as work performed under the auspices of the U.S. Department of Energy. 


\section{DISCLAIMER}

Portions of this document may be illegible in electronic image products. Images are produced from the best available original document. 


\title{
A proposed high-power UV industrial demonstration laser at CEBAF
}

\author{
Stephen V. Benson ${ }^{a}$, Joseph J. Bisognano ${ }^{a}$, Courtlandt L. Bohn ${ }^{\text {a }}$, Larry Cardman ${ }^{a}$, W. B. Colson ${ }^{b}$, \\ Paul Davidson $^{a}$, David Douglas ${ }^{a}$, H. F. Dylla ${ }^{a}$, David Engwallc, Jock Fugitta ${ }^{a}$, John Goldsteind, \\ Kevin Jordana, David Kehne ${ }^{a}$, Zhenghai Lie, Hongxiu Liua, Lia Merminga ${ }^{a}$, George R. Neila, \\ David Neuffer $^{\mathrm{a}}$, Michelle Shinn ${ }^{\mathrm{a}}$, Mark Wiseman ${ }^{\mathrm{a}}$, and Robert Wong ${ }^{\mathrm{b}}$
}

\author{
${ }^{a}$ Continuous Electron Beam Accelerator Facility, Newport News, VA 23606 USA \\ ${ }^{b}$ Naval Postgraduate School, Monterey, CA 93943 USA \\ ${ }^{c}$ Nuclear Physics Laboratory, Univ. of Ill. at Urbana-Champaign, Urbana, IL 61801 USA

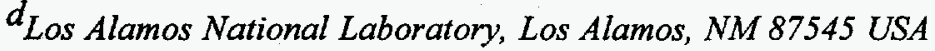 \\ ${ }^{e}$ Stanford Linear Accelerator Center, Stanford University, Stanford, CA 94305 USA
}

\begin{abstract}
The Laser Processing Consortium, a collaboration of industries, universities, and the Continuous Electron Beam Accelerator Facility (CEBAF) in Newport News, Virginia, has proposed building a demonstration industrial processing laser for surface treatment and micro-machining. The laser is a free-electron laser (FEL) with average power output exceeding $1 \mathrm{~kW}$ in the ultraviolet (UV). The design calls for a novel driver accelerator that recovers most of the energy of the exhaust electron beam to produce laser light with good wall-plug efficiency. The laser and accelerator design use technologies that are scalable to much higher power. We will describe the critical design issues in the laser such as the stability, power handling, and losses of the optical resonator, and the quality, power, and reliability of the electron beam. We will also describe the calculated laser performance. Finally progress to date on accelerator development and resonator modeling will be reported.
\end{abstract}

Key words: free-electron laser, UV laser, industrial laser, picosecond pulses

\section{Introduction}

In 1993 a collaboration of industries, universities, and the Continuous Electron Beam Accelerator Facility (CEBAF) in Newport News, Virginia, formed the Laser Processing Consortium with the purpose of using technologies developed at CEBAF to enhance the capabilities of U.S. industry. The Laser Processing Consortium's ultimate objective is to enhance U.S. industry's capability to profitably tailor the surface characteristics of polymers, composites, ceramics, and metals in an environmentally safe manner. To provide this capability, the consortium intends to develop a cost-effective, high-volume surface-processing system based on a high-average-power, wavelength-tunable free-electron laser (FEL). A companion paper gives examples of applications of the FEL [1].

Providing for industrial applications cost-effectively requires an average laser power of 50 to $100 \mathrm{~kW}$ delivered in 1 psec pulses at a cost below $\$ 0.01 / \mathrm{kJ}$. The laser must be continuously pulsed and should be tunable through wide portions of the UV and IR spectra where surface absorption is strong. The consortium proposed to develop this industrial capacity in two phases. Phase 1 entails construction of a UV/IR FEL demonstrator (hereafter called the UV Demo). The UV Demo, pictured in Figure 1, will provide $1 \mathrm{~kW}$ of average laser power with a continuously-pulsed, mode-locked time structure, at wavelengths between 190 and $300 \mathrm{~nm}$, with an option for IR operation between 3 and $20 \mu \mathrm{m}$. It will use technology that can be scaled up to meet industrial requirements. The UV Demo will enable validation of this technology, and industry will use this laser to develop and demonstrate processes of commercial interest. Experience gained from the UV Demo will be the basis for the Phase 2 laser. Phase 2 entails production of the full-scale $100 \mathrm{~kW}$ device and transfer of the technology to industry.

\section{UV FEL Capabilities}

An FEL works by extracting power from an electron beam via the mechanism of stimulated emission of bremsstrahlung in the sinusoidally varying magnetic field of a wiggle magnet [2]. Several features of the FEL combine to make it an attractive candidate for commercializing light-based manufacturing: 
- Average Power: Although not yet demonstrated, there is good reason to believe that FELs are capable of very high average power. The UV Demo will provide average powers in the kilowatt range. While it is straightforward to upgrade an FEL to higher power levels without increasing the cost substantially (decreasing the cost per kilojoule), existing sources in the UV increase in capital cost superlinearly with average power, so the cost per kilojoule remains high for high power systems.

- Tunability: Free-electron lasers are easily tunable by varying either the field strength in the wiggler or the electron beam energy according to the formula

$$
\begin{gathered}
\lambda_{\mathrm{r}}=\frac{\lambda_{\mathrm{W}}}{2 \gamma^{2}}\left(1+K^{2}\right) \\
K=\frac{\lambda_{\mathrm{W}} e B}{2 \pi m c^{2}},
\end{gathered}
$$

in which $B$ is the rms magnetic field of the wiggler, $\lambda_{\mathrm{r}}$ is the wavelength of laser radiation, $\lambda_{\mathrm{W}}$ is the wiggler wavelength, and $\gamma$ is the energy of the electrons divided by the rest mass energy of the electron $m c^{2}$.

The UV Demo will provide light that is tunable across most of the UV spectrum (190-300 nm) and, as an option, the IR spectrum ( 3 to $20 \mu \mathrm{m})$. As a bonus, the entire visible spectrum will be accessible $(350-750 \mathrm{~nm})$. This light will have all of the characteristics of high-quality laser emission: narrow bandwidth (typically $<0.1 \%$ ), spatial coherence (1-2 times the diffraction limit), and linear polarization.

- Temporal Structure: Existing lasers are either CW at low intensity or have pulses that are much too long to enable efficient surface processing. By contrast, FELs typically generate very short pulses $(\sim 1 \mathrm{psec})$ that are ideally suited to rapid thermal annealing or ablation of near-surface regions [3]. This pulse length matches the time scales of surface molecular rearrangements and vibrations. In ablation applications using high-power excimer lasers, pulse lengths exceed $10 \mathrm{nsec}$, and these pulses are long enough to interact with gas-phase ejecta with an associated loss of surface-interaction efficiency. The use of picosecond pulses avoids this problem.

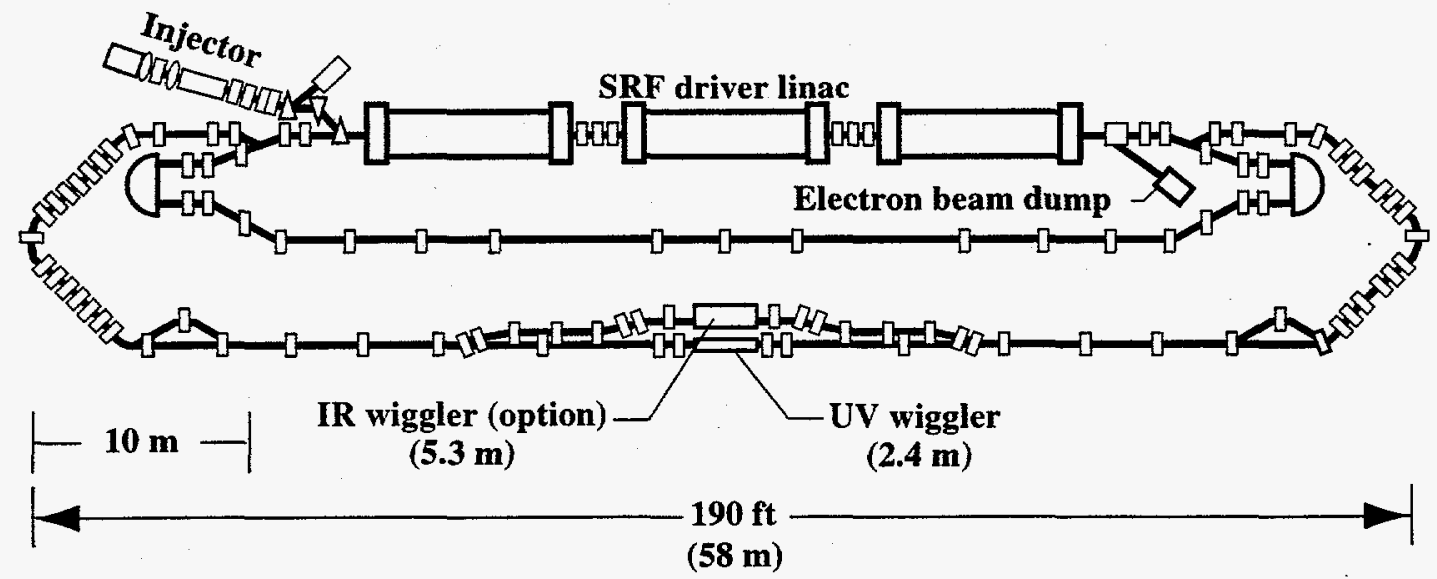

Figure 1. UV Demo schematic. The FEL extracts energy from electrons accelerated in two passes through an energy-recovering superconducting radio-frequency (SRF) driver linear accelerator (linac). A $10 \mathrm{MeV}$ electron beam from the injector attains $105 \mathrm{MeV}$ in the first of two passes of acceleration through the linac. After returning to the injection point clockwise through the low-energy recirculator line (at the center of the machine, between the linac and the wigglers), the beam attains $200 \mathrm{MeV}$ energy by the end of its second acceleration pass. Then it is steered through an FEL wiggler, where it yields about $0.5 \%$ of its power as laser light. Gain is produced in the wiggler via a coupling of the wiggler motion to the transverse field of the laser light. The exhaust electron beam then decelerates in two energy-recovery passes through the linac so that most of its energy can be recycled. Finally, the remaining electrons are absorbed when, decelerated to $10 \mathrm{MeV}$, they reach a cooled, shielded copper beam dump. 
- Efficiency and Cost: The cost per unit energy of light delivered from conventional UV sources is presently too high (of order $\$ 0.10 / \mathrm{kJ}$ ) for profitable industrial applications. For demonstration and development purposes, the UV Demo will provide light at about $\$ 1 / \mathrm{kJ}$. The cost of a free-electron laser does not scale strongly with the output power, however. Goals for the Phase 2 UV FEL are operation at $10 \%$ wall-plug efficiency and a cost of delivered light below $\$ 0.01 / \mathrm{kJ}$.

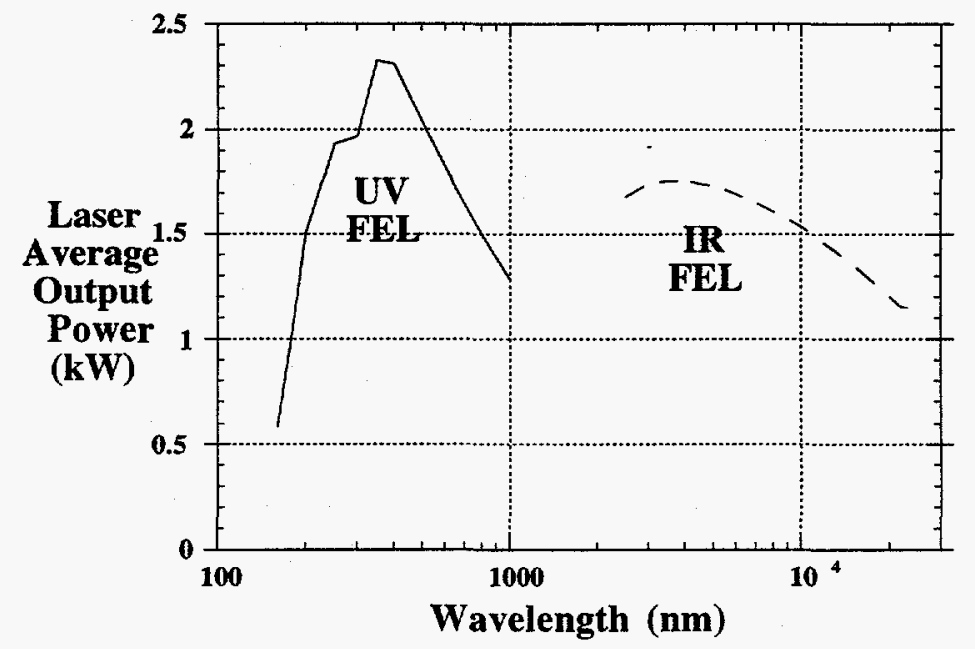

Figure 2. Power output versus wavelength calculated for the UV Demo and for the IR FEL option. The shortwavelength fall-off and kinks in the curve near 200 and $300 \mathrm{~nm}$ are due to changes in mirror technologies. The fall-off at long wavelengths is due to a lower electron beam energy leading to less available electron beam power.

The highest-priority considerations for the Phase 1 device are efficient, CW operation; low development cost; short development schedule; scalability; and high reliability, availability, and maintainability. All these will be even more important in the Phase 2 device. The Laser Processing Consortium accounted for all four considerations in formulating its development plan for the UV Demo.

One essential aspect of an industrial laser is energy recovery. During lasing, typically $<1 \%$ of the electrons' energy is converted to light. It is, however, possible to recover most of the energy in the spent beam and improve the overall efficiency. In the UV demo the electron beam is returned to the driver accelerator where it is decelerated, and most of its energy is converted back to radio-frequency (RF) power at the constituent cavities' resonant frequency. This recovered RF power is used to accelerate other electrons. The decelerated electrons are then deposited in a cooled, shielded beam dump. Energy recovery greatly reduces the accelerator's RF power requirements, waste heat, and radiation.

To enhance efficiency further, one can use superconducting radio-frequency (SRF) cavities. The very low wall loss in these cavities enables efficient $\mathrm{CW}$ operation. By contrast, room-temperature accelerators using copper cavities generally operate in a pulsed mode, producing typically $\sim 10 \mu \mathrm{sec}$ of beam several times a second. Accordingly, an SRF accelerator is essential to high average power and energy efficiency in $\mathrm{CW}$ acceleration and deceleration of the electron beam. Moreover, SRF technology is well suited for transporting high-current beams while preserving high beam quality at the entrance to the FEL wiggler, and thereby ensuring efficient conversion of electron beam energy into light. Finally, the technology can be scaled up for use in Phase 2.

\section{UV Demo Design}

The user requirements and the design goals of the UV demo laser are shown in Table 1 [4]. The design goals exceed the user requirements to provide a safety margin. To minimize cost and schedule, existing designs or commercially available components are incorporated into the UV Demo wherever possible. Many of these existing designs derive from CEBAF's $4 \mathrm{GeV}$ recirculating SRF accelerator, which began operation for nuclear physics research in 1995 . The injector consists of a $500 \mathrm{keV}$ DC 
photocathode gun driven by a commercially available Nd:YLF laser, followed by a copper cavity to bunch the beam and a CEBAF-type SRF quarter-cryomodule operating at $1500 \mathrm{MHz}$ to provide acceleration to $10 \mathrm{MeV}$. The driver accelerator uses CEBAF SRF cavities operating at $1500 \mathrm{MHz}$ and producing $32 \mathrm{MV}$ per eight-cavity cryomodule. The UV Demo also incorporates a commercially available wiggler and copies of CEBAF's RF system, control system, and safety system.

Table 1. User requirements and design goals for the UV Demo

\begin{tabular}{|c|l|l|}
\hline & User Requirements & Design Goal \\
\hline $\begin{array}{c}\text { Wavelength range } \\
\text { UV }\end{array}$ & $190-300 \mathrm{~nm}$ & $160-1000 \mathrm{~nm}$ \\
IR & $3000-20,000 \mathrm{~nm}$ & $2500-25,000 \mathrm{~nm}$ \\
\hline Power & $1 \mathrm{~kW}$ & $1.7 \mathrm{~kW}$ \\
\hline Optical beam quality & $<2 \times$ diffraction limit & $1.2 \times$ diffraction limit \\
\hline Bandwidth & Within $4 \times$ Fourier limit & Fourier limited \\
\hline Stability & & \\
Temporal & $<25 \%$ & $<10 \% \Delta P / P$ in 30 min. \\
Operational & $<1$ trip every 10 min. & $<1$ trip every hour \\
Wavelength & $<10^{-2} \Delta \lambda / \lambda$ & $<2 \times 10^{-3} \Delta \lambda / \lambda$ \\
Pointing & $<10 \mu \mathrm{rad}$ & $<1 \mu \mathrm{rad}$ \\
\hline Runtime & $>2.5$ hours (gun limited) & Continuous, 1000 hours \\
\hline Energy recovery & $>90 \%$ & $95 \%$ \\
\hline
\end{tabular}

The use of CEBAF cryomodules also provides confidence that the reliability, availability, and maintainability of the UV Demo will be high, based on CEBAF's experience with building, installing, and operating the $421 / 4$ cryomodules in the existing accelerator [5]. A related requirement is that electron beam impingement on the walls of the device be kept low $(<5 \mu \mathrm{A}$ at $>25 \mathrm{MeV}$ ) to reduce radiation damage, radiation-shielding requirements, and spurious electronic noise. Low beam loss-which is an attribute of the intrinsic large aperture of SRF cavities-also contributes to safe hands-on maintenance of the device. Otherwise, over time, radioactivation of components due to beam impingement could conceivably become a problem.

As noted above, the wavelength of the FEL is determined by the wiggler wavelength, the electron beam energy and the wiggler strength, represented by the wiggler parameter $K$. Significant laser gain requires $K \sim 1$. Although it is certainly possible to produce a wiggler period as short as $1 \mathrm{~cm}$ with $K \sim 1$, the resulting wiggler gap is quite small and the available electron beam power is reduced due to the lower electron beam energy. We have therefore chosen a wiggler with a period of $3.3 \mathrm{~cm}$ which allows a $1 \mathrm{~cm}$ clear vertical aperture for the electron beam and optical mode. This leads to the need for a $200 \mathrm{MeV}$ electron beam to generate a $190 \mathrm{~nm}$ wavelength.

Experience with other FELs (confirmed by detailed modeling) suggests that the conversion efficiency of the FEL is $\sim\left(4 N_{\mathrm{W}}\right)^{-1}$, where $N_{\mathrm{W}}$ is the number of wiggler periods. To obtain sufficient gain at UV wavelengths requires $N_{\mathrm{W}} \sim 70$, corresponding to $0.35 \%$ conversion of electron beam energy to light. Allowing for the significant $(-50 \%$ net) losses in commercially available optics at the shortest wavelengths, the electron beam power must be $1 \mathrm{MW}$ to obtain a laser power of $1.75 \mathrm{~kW}$. Thus, the average beam current must be $I_{\text {avg }} \sim 5 \mathrm{~mA}$.

It is possible to estimate limits on the electron beam's energy spread and transverse emittance as well. The total phase slip in the wiggler is $N_{\mathrm{W}}$. If the energy spread is greater than $\left(5 N_{\mathrm{W}}\right)^{-1}$, then the electrons will dephase by a full optical period with respect to the optical field while traversing the wiggler. To avoid significant reduction in gain, one must therefore keep the fractional rms energy spread within $2.8 \times 10^{-3}$. To ensure transverse overlap of the electron beam with the optical mode, the normalized beam emittance must be $\sim \gamma \lambda_{r} /(4 \pi) \pi$-mm-mrad. This is a difficult condition to meet and it cannot be grossly violated, but a factor-of-two violation does not unduly degrade the beam-mode coupling. This means the normalized transverse emittance $\left(\varepsilon_{n}\right)$ must be within approximately $12 \pi$-mm-mrad. 
We have found several areas that introduce risk into the accelerator design.

- One must achieve high brightness with a continuously pulsed, high-charge electron beam.

- Emittance growth in such a machine must be carefully controlled.

- An accelerator with a net synchrotron tune of one-half is inherently unstable and must have active feedback control [6].

- Beam losses in a high-power machine must be kept very small both for machine protection and to ensure RF stability.

- Mirror coatings in the deep UV are lossy. This leads to problems with poor output coupling and mirror distortion.

We also considered other limits that might affect FEL performance. In all other cases, either the FEL performance was insensitive to the parameter or the parameter was easily achieved.

The sensitivity of the laser performance with respect to these parameters is apparent by way of the following scaling relationship for the laser power:

$$
P_{\text {laser }} \cong \frac{E_{\mathrm{b}} I_{\mathrm{avg}}}{4 N_{\mathrm{W}}} \frac{E_{\mathrm{b}} \lambda_{\mathrm{r}}}{4 \pi \varepsilon_{\mathrm{n}} m c^{2}}\left(1-\delta-\frac{3 \delta}{G_{\mathrm{ss}}}\right)
$$

where $\delta$ represents the non-output-coupling losses (absorption and scatter from the mirrors, and diffraction from apertures), and $G_{\mathrm{ss}}$ is the laser gain at small signal. This relationship includes several simplifying approximations, yet detailed modeling shows that it is a good qualitative representation of the scaling of laser power with parameters associated with the electron beam and photon optics.

The laser power is clearly seen to be most sensitive to the electron beam energy $E_{\mathrm{b}}$. The average current, normalized transverse emittance, and mirror losses are also critical design parameters. The dependence on the energy spread is hidden in $G_{\text {ss }}$ and is relatively weak. The dependence on $N_{\mathrm{W}}$ is also relatively weak because $G_{\mathrm{ss}}$ varies linearly with $N_{\mathrm{W}}$, but the efficiency is inversely dependent on $N_{\mathrm{W}}$, so the net power dependence is fairly flat. The dependence on peak current is stronger because $G_{s s}$ also varies linearly with peak current, and so the peak current should be as high as possible. Modeling indicates that the electron beam bunches can be made short enough to produce a peak current $I_{\mathrm{pk}}=270 \mathrm{~A}$.

One critical parameter not reflected in the scaling relationship is mirror deformation. Detailed modeling of various shortwavelength lasers indicates that laser power is not significantly degraded as long as the mirrors do not deform by more than $1 / 5$ of an optical wave. Detailed analysis of the optical resonator for the UV Demo remains to be done, but the specification is believed to be similar or more relaxed. This is still an extremely tight tolerance at $200 \mathrm{~nm}$, especially with high power loading, and active figure control may be required.

The critical electron beam design values chosen for the conceptual design of the UV Demo after detailed analysis are listed in Table 2 with respect to a nominal laser wavelength $\lambda_{\mathrm{r}}=200 \mathrm{~nm}$. Sensitivities of the laser gain and output power with respect to variations in these critical parameters about their design values $\left(\lambda_{\mathrm{T}}=200 \mathrm{~nm}\right)$ are illustrated in Table 3 . Our best estimates of the largest possible degradation are assumed in calculating the performance degradation in the laser. We have found some evidence that coherent synchrotron radiation in the bending magnets of the driver accelerator may lead to a growth in the emittance larger than the estimate given in Table 3. This phenomenon is poorly understood, but since the emittance is a critical parameter, we are trying to better understand the effect and come up with both better estimates of its size and ways to alleviate the problem [7].

As noted previously, energy recovery is an essential aspect of the UV Demo design and must be demonstrated for the Phase 2 device to be realizable. Another important aspect is multipass acceleration (recirculation). With recirculation, the machine generates the required beam energy at the wiggler with a shorter accelerator. Since costs of the cryomodule and associated RF powers dominate the cost of the accelerator, a multipass machine is considerably less expensive than a single-pass machine. To produce a $200-\mathrm{MeV}$ beam with a $32-\mathrm{MeV}$ CEBAF cryomodules preceded by a $10 \mathrm{MeV}$ injector, there are several 
options: three passes with two modules, two passes with three modules, six passes with one module, or one pass with six modules. The last option is too costly, and a six-pass machine was deemed too complex. The choice between two passes versus three passes was slightly in favor of the two-pass machine based on reduced complexity and better preservation of beam quality. Thus, the conceptual design of the UV Demo consists of a $10 \mathrm{MeV}$ injector feeding into a two-pass recirculating accelerator comprising three CEBAF cryomodules.

Table 2. Critical electron beam design values for the UV Demo

\begin{tabular}{|l|l|}
\hline Electron beam energy & $E_{\mathrm{b}}=200 \mathrm{MeV}$ \\
\hline Average current & $I_{\mathrm{avg}}=5 \mathrm{~mA}$ \\
\hline Peak current & $I_{\mathrm{pk}}=270 \mathrm{~A}$ \\
\hline Normalized transverse emittance & $\varepsilon_{\mathrm{n}}=11 \pi$-mm-mrad \\
\hline Non-output-coupling loss & $\delta=8 \%$ \\
\hline Mirror deformation & $40 \mathrm{~nm}$ \\
\hline
\end{tabular}

Table 3. Sensitivities of laser gain and power versus degradation in critical parameters for $200 \mathrm{~nm}$ operation

\begin{tabular}{|l|c|c|c|}
\hline & & \multicolumn{2}{|c|}{ Impact on Power and Gain } \\
\hline Parameter & Possible degradation & Power & Gain \\
\hline Energy & $-10 \%$ & $32 \%$ & $25 \%$ \\
\hline Transverse emittance & $+25 \%$ & $38 \%$ & $30 \%$ \\
\hline Energy spread & $+33 \%$ & $12 \%$ & $10 \%$ \\
\hline Peak current & $-25 \%$ & $15 \%$ & $23 \%$ \\
\hline Optical cavity losses & $+25 \%$ & $13 \%$ & 0 \\
\hline
\end{tabular}

A user facility will be constructed on the CEBAF site to house the UV Demo and provide laboratories in which to demonstrate and develop laser-processing applications. The UV Demo will be housed below grade level on the first floor, a "rigid-box" concrete structure that will "float" on compacted soil for stability. The laboratory space on the second floor, a steel-framed structure including areas for housing RF power, electronics, and controls, can be configured into six or more user areas served by optical beamlines from the FEL below. There is substantial room for laser-applications work, with low-conductivity cooling water, dry gas, electrical services, chemical hoods, and exhaust. Quick vacuum interconnects will permit enclosure of the optical beam and transport to any of the areas, and remotely insertable mirrors will allow delivery of the beam to any station desired. All areas will be networked to the computer systems controlling the laser. Shielding between floors will reduce radiation hazards to acceptably low levels typical of laboratory work.

\section{Critical Technical Issues}

During the design process we identified several critical technical issues. The design was iterated so that the reduction in performance due to each technical risk area was similar. In this way the risk is evenly spread among all possible risk factors. In each case there are means to recover from shortfalls in performance. The development plan arrived at for building the UV Demo is focused on mitigating the critical technical issues.

\subsection{Injector}

No injector exists which will provide the required combination of continuous average current and emittance. Space-charge effects in the high-current beam are important. If necessary, the injector can be operated at twice the planned pulse-repetition rate and half the charge per bunch to reduce space-charge effects. If cathode lifetime becomes a limiting factor, an alternative cathode can be used.

An injector test stand is already under construction at CEBAF to test the injector design [8]. A shielded enclosure has been modified to accept the injector. Several subsystems such as the photocathode drive laser and the high-voltage power supply have been constructed and tested. Components for the HV gun are almost complete and assembly has begun. The quarter- 
cryomodule and RF systems are scheduled to be completed by spring of 1996. Prototypes of non-standard components for this quarter-cryomodule have been fabricated and successfully tested. The performance of CEBAF cavities in the presence of high beam loading will be measured during injector tests. The emittance and charge-density profile of the beam will be carefully measured, and the results will be correlated with analytic theory and numerical simulations.

\subsection{Energy Recovery}

The basic principles of energy recovery have been demonstrated experimentally [9], but energy recovery at high average beam current with an FEL increasing the energy spread of the exhaust beam has not been demonstrated. Transport must be nearly lossless from $\sim 199 \mathrm{MeV}$ down to the beam dump at $10 \mathrm{MeV}$. Recirculating, energy-recovering accelerators exhibit instabilities that arise from fluctuations of the cavity fields. Energy changes can cause beam loss on apertures, or, when coupled to energy dependent timing, phase oscillations. Both effects change the beam induced voltage in the cavities and can lead to unstable variations of the accelerating field. Stability analysis for small perturbations from equilibrium has been performed [6] and the threshold current for the CEBAF FEL has been determined to be $300 \mu \mathrm{A}$. The model has been extended to include amplitude and phase feedback, with the transfer function of the feedback presently modeled as a low-pass filter. It was found that, for small variations, modest gain frequencies, well within CEBAF's RF control system capability, are required to stabilize the system.

Instabilities may arise, and the RF control system may need to incorporate additional fast-feedback capability to damp them. An alternative lattice has been developed which has small net beam loading, and it could be incorporated should the baseline control system prove insufficiently responsive to transients. It has the disadvantage of reduced energy acceptance in the first recirculation pass, but it should be absolutely stable. Beam loss must be kept low to reduce radiation damage, shielding requirements, spurious electronic noise, and radioactivation of components. To mitigate beam loss, a few specific apertures could be increased, and local shielding and beam scrapers could be inserted at strategic locations around the machine.

Until actual construction and commissioning of the UV Demo, analysis of energy recovery will continue. Modeling of the system performed to date incorporates known effects, including ongoing analysis of transients related to turn-on of the FEL.

\subsection{Optical Cavity}

To handle the high average power density in the UV Demo with reasonable mode quality, we plan to use a retro-reflecting, re-imaged, ring resonator (or R5 resonator) [10]. The R5 resonator is a negative-branch, unstable resonator with scraper output coupling. This design has the advantage of using all reflective surfaces. The lowest-order mode of this resonator has a top-hat profile at the scraper and an Airy profile at the wiggler (the Airy profile is the two-dimensional Fourier transform of the uniform disk). Simulations both with a Beer's law gain profile (the gain was exponential in the distance along the wiggler and had a Gaussian transverse profile with the same size as the electron beam) and with the FEL 3D simulation code FELEX [11] show that the presence of the wiggler bore and a gain medium rounds the top-hat profile and reduces the output coupling for a given magnification. The gain of this mode is less than the gain of a Gaussian mode. Gaussian mode simulations at $200 \mathrm{~nm}$ predict a gain of $125 \%$ while FELEX predicts approximately $100 \%$. The Gaussian simulation predicts a peak power output of $130 \mathrm{MW}$ while the FELEX simulation predicts a power output of $70 \mathrm{MW}$. Given a duty cycle of $1.8 \times 10^{-5}$, the average power is greater than $1300 \mathrm{~W}$. An interesting feature of the FELEX simulations is that the power output is insensitive to the electron beam focusing, contrary to expectations.

A serious disadvantage of the R5 resonator design is the need for six reflections in each round trip. Though mirrors are quite adequate for wavelengths longer than $250 \mathrm{~nm}$, the reflectivity of mirrors in the deep UV is at best $98-99 \%$ for normal incidence and is usually only about $92 \%$ for a $54^{\circ}$ p-plane reflection. We plan to work with the University of Arizona Optical Science Center to develop deep UV optics with higher p-plane reflectivity. If this does not work, we will use a roof-top reflector with all s-plane reflections, which is quite adequate for the kilowatt demonstrator but is not scalable to the $100 \mathrm{~kW}$ device.

To test such issues as depolarization, net reflectivity, length control, and figure control, we are building a full-size mockup of the R5 resonator using mirrors with reflective coatings at $633 \mathrm{~nm}$. This will allow us to test the basic design of the resonator before building the final device.

High-power laser operation will result in a significant heat load $\left(\sim 50 \mathrm{~W} / \mathrm{cm}^{2}\right)$ on the mirrors, making it difficult to limit mirror deformation. Moreover, losses in commercially available UV optical coatings increase significantly below $225 \mathrm{~nm}$. Improved optical coatings for the shortest wavelengths are being developed in collaboration with industrial and university labora- 
tories that have established reputations in deep-UV optics: An alternative optical cavity which uses commercially available coatings has been developed as a backup. This cavity fulfills the requirements for the UV Demo, but it has limited scalability to higher power. To mitigate mirror deformation, the substrates can be cooled to liquid-nitrogen temperatures where the coefficient of expansion approaches zero. Alternatively, the cavity can be made longer.

The optical system requires development of an advanced mounting, mirror substrates, and optical controls and diagnostics. An optical test stand will be constructed early in the project and subsequently used to validate the various components of the optical cavity. A special optical test bed at the National Institute of Standards and Technology will be used for absorption and scattering measurements of optical coatings prior to their installation on the UV Demo.

\section{Conclusion}

In summary, industry has significant need for the development of a cost-effective, high-power UV/IR FEL for surface-processing applications. In response, the Laser Processing Consortium formed, and it has proposed a systematic program culminating in the desired machine. The program should proceed in two phases, Phase 1 culminating in the $1 \mathrm{~kW}$ UV Demo, and Phase 2 culminating in the full-scale UV/IR FEL. No show-stoppers are foreseen. The proposed plan is a responsible development path that should allow technical approaches to be demonstrated at $1 \mathrm{~kW}$ before the scale-up to an industrial $50-100 \mathrm{~kW}$ system.

Optimization studies for the UV Demo have produced a robust design that meets the minimum requirements of the users. Several potential problems have been extensively studied and found soluble. We are continuing the study of the emittance growth of short-pulse electron beams, which might be worsened by coherent synchrotron emission [7], and the study of the overall phase and gradient stability of energy-recovered systems. The lattice is also being optimized to reduce delivery time and cost.

We have recently received approval for a kilowatt demonstrator in the infrared. This device is to be built in support of a program of ship defense sponsored by the Office of Naval Research. It is a useful first step in the Phase I plan since the injector, energy recovery, and emittance preservation can all be tested in the planned device for one-third the cost of the Phase I device. The IR FEL will use the same injector as the UV FEL but will only use a single acceleration pass through a single cryomodule. The final energy will therefore be $42 \mathrm{MeV}$ instead of $200 \mathrm{MeV}$. The peak current will also be at least a factor of five lower than in the UV device, and the emittance constraint is slightly relaxed. The wiggler will be a small fixed-gap commercial wiggler with a wiggler period of approximately $2 \mathrm{~cm}$. This will provide $1 \mathrm{~kW}$ of power near $2 \mu \mathrm{m}$. The optical resonator will be a nearly concentric design with dielectric mirrors.

\section{Acknowledgments}

This work was supported by the Commonwealth of Virginia, the Virginia Center for Innovative Technology, and DOE Contract \# DE-AC05-84ER40150.

\section{References}

1. M. Kelley, "UVFEL light source for industrial processing" SPIE conference proceedings Vol 2703A

2. For a good description of the physics of free-electron lasers, see C. A. Brau, Free-Electron Lasers (Academic Press, San Diego Ca, 1990). or M. L. Stitch, M. S. Bass, Eds., Laser Handbook, vol. 4 (North Holland, Amsterdam, 1985).

3. S. V. Nair, "Applications of Free-electron Lasers to Metals and Ceramics processing", CEBAF Tech. Note TN94057 (1994).

4. Laser Processing Consortium "Free-Electron Lasers for Industry", available from CEBAF, Newport News VA and H. F. Dylla, "A High-Average Power FEL for Industiral Applications", Proc. 1995 Particle Accelerator Conf. Dallas Texas (1995).

5. C. Reece, J. Benesch, M. Drury, C. Hovater, J. Mammosser, T. Powers, J. Preble, "Performance Experience with the CEBAF SRF Cavities", Proc. 1995 Particle Accelerator Conf. Dallas Texas (1995). 
6. L. Merminga, J. J. Bisognano, J. R. Delayen, "Energy Stability in Recirculating, Energy-recovering Linacs," presented at Free-electron Lasers '95, New York, NY, Aug. 1995. 7. C. L. Bohn, Proc. Microbunches Workshop, Brookhaven National Laboratory, 28-30 Sept. 1995 (in
publication).

8. H. Liu et al., "Design of a high charge CW photocathode injector test stand at CEBAF", Proc. 1995 Particle Accelerator Conf. Dallas Texas (1995).

9. T. I. Smith, H. A. Schwettman, R. Rohatgii, Y. LaPierre, and J. Edighoffer, Nucl. Inst. and Meth. A259 (1987) 1-7. and D. W. Feldman et al., Nucl. Inst. and Meth. A259 (1987) 26-30.

10. Chun-Ching Shih and Su-Miau Shih, Nucl. Inst and Meth. A304 (1991) 788.

11. B. D. McVey, Nucl. Inst. and Meth. A250 (1986) 449. J. C. Goldstein et al., Proc. SPIE 1045, "Modeling and Simulation of Laser Systems", ed. B. L. Bullock (1989) p. 28.

\section{DISCLAIMER}

This report was prepared as an account of work sponsored by an agency of the United States This the United States Government nor any agency thereof, nor any of their employees, makes any warranty, express or implied, or assumes any legal liability or responsibility for the accuracy, completeness, or usefulness of any information, apparatus, product, or process disclosed, or represents that its use would not infringe privately owned rights. Referprocess disclose manufacturer, or otherwise does not necessarily constitute or imply its endorsement, recommendation, or favoring by the United States Government or any agency thereof. The views and opinions of authors expressed herein do not necessarily state or reflect those of the United States Government or any agency thereof. 